\title{
EFECTO DE UNA BACTERINA DE DOSIS ÚNICA CONTRA Mycoplasma hyopneumoniae SOBRE EL TÍTULO DE ANTICUERPOS, GANANCIA DE PESO Y LESIONES PULMONARES EN PORCINOS PROVENIENTES DE MADRES VACUNADAS
}

\author{
Chris Pinto J. ${ }^{\text {, }}$, Sonia Calle E. ${ }^{1,2}$, Marlon Torres A. ${ }^{3}$, César Gavidia Ch. ${ }^{4}$, \\ Néstor Falcón P. ${ }^{4}$, Francisco Acosta Ch. ${ }^{5}$ y Siever Morales C. ${ }^{1}$
}

\section{Abstract}

The aim of the present study was to determine whether the immunization with a single dose of a commercial bacterin against Mycoplasma hyopneumoniae influences antibody titles, body weight gain, and lung lesions in a commercial pig farm. Sixty piglets born from vaccinated sows were used. One group of 30 piglets ( 15 males and 15 females) were vaccinated at 42 days of age and the other group remained unvaccinated, as a control group. Blood samples were collected at 21, 42, 70, 84, 112, and 145 days of age to determine antibody titles with an Indirect ELISA test. Animals were weighed at 21 and 145 days of age and lung lesions were evaluated at slaughter (145 days). Nearly $60 \%$ of pigs from both groups had high antibody titres at 21 days of age, but decreased by day 70 . Titres increased between 84 and 145 days of age in the vaccinated group while increased by day 112 in the unvaccinated group. There were no significant differences in body weight gain between both groups. The 79.3 and $20.7 \%$ of piglets from the control group and the vaccinated group respectively, presented lung lesions. The results showed that vaccination with a single dose bacterin against $M$. hyopneumoniae increased antibody titles, although not in the entire population, and diminished the frequency of lung lesions, but no influenced body weight gain.

Key words: Mycoplasma hyopneumoniae, pig, one dose bacterin, antibody title, body weight gain

\section{Resumen}

El objetivo del presente estudio fue determinar si la inmunización con bacterina de dosis única contra Mycoplasma hyopneumoniae afecta los títulos de anticuerpos, mejora la ganancia de peso, y disminuye las lesiones pulmonares en porcinos de crianza intensiva. Se emplearon 60 lechones provenientes de madres vacunadas, los cuales fueron divididos en 2 grupos de 30 lechones ( 15 hembras y 15 machos). Un grupo fue inmunizado a los 42 días de edad y el otro fue utilizado como control. Se tomaron muestras de sangre a los 21, 42, 70, 84, 112 y 145 días para determinar el título de anticuerpos con la prueba de ELISA indirecta. Los lechones se pesaron a los 21 y 145 días de edad y

\footnotetext{
${ }^{1}$ Laboratorio de Microbiología y Parasitología Veterinaria, FMV-UNMSM

${ }^{2}$ E-mail: calleson@gmail.com

${ }^{3}$ Laboratorio de Producción Agropecuaria, FMV-UNMSM

${ }^{4}$ Laboratorio de Epidemiología Veterinaria Preventiva, FMV-UNMSM

${ }^{5}$ Práctica privada
} 
las lesiones pulmonares se evaluaron al beneficio (145 días). Cerca del $60 \%$ de porcinos en ambos grupos presentaron títulos altos de anticuerpos a los 21 días de edad, disminuyendo hacia los 70 días. El grupo inmunizado elevó sus títulos entre los 84 y 145 días de edad y el grupo control los elevó hacia los 112 días de edad. No existió diferencia estadística significativa en la ganancia de peso de ambos grupos. El 79.3 (23/29) y $20.7 \%$ (6/29) del grupo control e inmunizado, respectivamente, presentaron lesiones pulmonares al beneficio. Se puede concluir que la inmunización con bacterina de dosis única contra $M$. hyopneumoniae elevó los títulos de anticuerpos de los porcinos inmunizados en gran parte de la población, y disminuyó el porcentaje de lesiones pulmonares al beneficio, pero no influyó en la ganancia de peso.

Palabras clave: Mycoplasma hyopneumoniae, porcino, bacterina de dosis única, título de anticuerpos, ganancia de peso

\section{INTRODUCCIÓN}

Actualmente, la industria porcina enfrenta una serie de retos y cambios en su estructura en búsqueda del mejoramiento productivo. Esto ha llevado a los productores a desarrollar sistemas de producción que disminuyan las posibilidades de infección con diversos patógenos, y así, minimizar las pérdidas económicas derivadas por las mermas productivas.

\section{Entre los patógenos comúnmente cono- cidos que residen en el tracto respiratorio, $\mathrm{d}$} Mycoplasma hyopneumoniae es el agente causal de un cuadro neumónico denominado Neumonía Micoplásmica (NM) (Ross, 2000). Esta enfermedad ocasiona serias pérdidas económicas en la industria porcina alrededor del mundo, ya que disminuye el promedio de ganancia de peso y la eficiencia de conversión, produce desigualdad en los lotes y prolonga los días de salida al mercado (Valdivia, 1999). Además, es el agente primario del Complejo Respiratorio Porcino (CERP) (Piffer y Ross, 1984), que predispone a los porcinos a infecciones secundarias como las producidas por Actinobacillus pleuroneumoniae (Yagihashi et al., 1984), Haemophilus parasuis y Pasteurella multocida (Morrison et al., 1985) y algunos virus, entre los que se encuentran el del Síndrome Respiratorio Reproductivo Porcino (SRRP) (Thacker et al., 1999), Influenza Porcina (IP) y Circovirus tipo II.
La inmunización contra $M$. hyopneumoniae es una estrategia sanitaria que usualmente se realiza con bacterinas de dos dosis. El inconveniente de ese tipo de vacunas es el estrés producido en los animales durante la manipulación de la aplicación de la vacuna, de allí la conveniencia de evaluar la efectividad de la inmunización con una bacterina de dosis única.

\section{Materiales y Métodos}

\section{Lugar de estudio}

El trabajo se realizó durante los meses de marzo a agosto del 2004 en una granja porcina tecnificada, positiva a Mycoplasma hyopneumoniae, ubicada en el distrito de Puente Piedra, Lima. En la granja se practica el sistema de crianza "todo dentro todo fuera" con producción en un solo sitio, posee un plantel reproductor de 600 vientres de un cruce para línea materna, no practica la inmunización contra $M$. hyopneumoniae y el alimento suministrado no contiene antibióticos contra micoplasma.

\section{Animales}

Se utilizaron lechones provenientes de madres multíparas (3-4 partos) en buen estado de salud, que se les inmunizó contra $M$. hyopneumoniae a los 85 días de gestación (bacterina de los Laboratorios Pfizer). El 
tamaño muestral se definió mediante la fórmula de "Comparación de medias" (Snedecor y Cochran, 1986).

Los lechones seleccionados fueron distribuidos en forma uniforme de acuerdo a su tamaño, pero manteniéndolos en el mismo lote y cuarto de maternidad, con animales de su misma edad. Fueron divididos en 2 grupos de 30 lechones (15 hembras y 15 machos) donde al primero se le aplicó $2 \mathrm{ml}$ de una bacterina contra M. hyopneumoniae (Laboratorios Pfizer) a los 42 días de edad y el otro grupo quedó como control, aplicándosele una solución salina estéril al $0.09 \%$ para simular el estrés de la manipulación en la vacunación.

\section{Muestras de sangre}

Se recolectaron muestras de sangre de la vena cava craneal a los $21,42,70,84,112$ y 145 días de edad, periodo que comprende desde el destete al beneficio. El suero se extrajo por centrifugación a $3000 \mathrm{rpm}$ durante $10 \mathrm{mi}$ nutos y fue conservado a $-20{ }^{\circ} \mathrm{C}$. Los sueros se analizaron mediante la técnica de ELISA indirecta con el kit comercial de ELISA HerdChek* M hyo (Laboratorios Idexx). Las muestras de suero se procesaron en el Laboratorio de Bacteriología de la Facultad de Medicina Veterinaria de la Universidad $\mathrm{Na}$ cional Mayor de San Marcos.

\section{Ganancia de peso}

Los lechones se pesaron a los 21 y 145 días de edad, y la ganancia de peso se determinó por la diferencia entre los dos pesos.

\section{Consolidación pulmonar}

Se evaluó el grado de lesión pulmonar en los animales beneficiados. Se examinaron los 7 lóbulos de cada pulmón, asignándoles una puntuación a cada lóbulo de acuerdo al porcentaje de consolidación pulmonar (Piffer y Britto, 1991). Para esto, se mide la extensión de la lesión de consolidación pulmonar en cada lóbulo (expresada en \%) y se calcula el área pulmonar consolidada por lóbulo, tomando en cuenta su peso relativo, y se suma cada parte para obtener el área pulmonar consolidada total. El área de consolidación se agrupó en 6 categorías, siguiendo el modelo propuesto por Piffer y Britto (1991).

\section{Análisis estadístico}

Los títulos de anticuerpos fueron analizados con el programa estadístico Stata 8.0 usando la prueba de Kolmogorov Smirnov de dos muestras, ante la falta de normalidad para analizar diferencias estadísticas entre los grupos.

En la evaluación del peso y la ganancia de peso se usó la prueba de análisis de varianza por bloques, donde se bloqueó la variable sexo. Las lesiones pulmonares se analizaron con la prueba de Chi Cuadrado para determinar la posible asociación entre el porcentaje de consolidación pulmonar y la vacunación.

\section{Resultados y Discusión}

El porcentaje de seropositividad a los 21 días de edad a $M$. hyopneumoniae, debido a la transferencia de anticuerpos maternales, fue similar para ambos grupos (56.7 y $60.0 \%$ para el grupo inmunizado y el grupo control, respectivamente).

La respuesta inmune a la vacunación puede alterarse cuando se realiza entre las 2 y 4 semanas de edad, o cuando se tienen niveles elevados de anticuerpos (Thacker et al., 1998; Jayappa et al., 2001), por lo que se prefiere hacerlo en una edad más avanzada (Quinlan, 1998; Thacker, 2001). A su vez, la influencia de los anticuerpos maternales puede variar entre granjas (Jensen et al., 2000; Pommier et al., 2000; Thacker et al., 2000).

El promedio de los títulos de anticuerpos en los seis muestreos del grupo inmunizado y del grupo control fue de 3.06 y 3.21 , respectivamente, no habiendo diferencia estadística significativa entre ellos (Fig. 1, p>0.05). 
El porcentaje de animales seropositivos previo a la vacunación (21 y 42 días de edad) fue similar en ambos grupos. En cambio, se obtuvo un $30 \%(9 / 30)$ de animales seropositivos a los 70 días de edad en contraste con el 3.3\% (1/30) en los animales control (Fig. 2), con diferencias estadísticas en los títulos de anticuerpos (2.72 vs 2.0, respectivamente, Fig. 1). El porcentaje de lechones seropositivos se incrementó en los muestreos a los 84 y 112 días de edad (53.3 y $56.7 \%$, respectivamente), en tanto que la frecuencia del grupo control subió ligeramente a los 112 días (10.3\%) (Fig. 2). Las diferencias estadísticas entre los grupos inmunizados y control se mantuvieron hasta los 112 días de edad $(\mathrm{p}<0.05)$. Estas diferencias se debieron a la respuesta a la vacunación en el grupo inmunizado y a la caída de los anticuerpos maternales en el grupo control. Es posible que los cerdos control se hayan infectado entre los 70 y 84 días de edad, de allí que se observara seroconversión en los muestreos siguientes.

Es importante resaltar que los anticuerpos maternales de la población deben estar disminuidos para que se pueda presentar la infección (Torres, 2003) y que la seroconversión ocurra a las 4 semanas de exposición al patógeno (Clark, 1999). Los resultados concuerdan con otros estudios (Clark, 1999; Joo et al., 1988; Blood et al., 1999), aunque difieren con las infecciones más tempranas reportadas por Torres (2003). Por otro lado, se conoce que la seroconversión ante infecciones naturales es más lenta que como resultado de infecciones experimentales (Calsamiglia et al., 1999) y que se encuentra afectada por la composición genética de los animales (Goodwin, 1985).

El sexo de los lechones no afectó significativamente $(p>0.05)$ la frecuencia de seropositivos ni los valores de anticuerpos, tal y como fue demostrado por este equipo de investigadores en trabajos previos (Calle et al., 2003).
En el análisis de la ganancia de peso se excluyó un animal por grupo, debido a que murieron por causas ajenas al estudio a los 102 y 137 días. No se encontró diferencia estadística significativa entre grupos ( $p>0.05)$, donde el grupo inmunizado tuvo una ganancia de peso de $82.5 \mathrm{~kg}$ frente a los $82.9 \mathrm{~kg}$ del grupo control. Tampoco hubo diferencias entre grupos y por sexos, aunque se pudo observar una mayor uniformidad en la ganancia de peso de los machos inmunizados. No obstante, en el análisis global, los machos obtuvieron una ganancia de peso mayor que las hembras ( 88.0 vs. $77.4 \mathrm{~kg}$, respectivamente, $\mathrm{p}<0.05)$.

Por otro lado, se tiene evidencia, a través de un metanálisis de 41 estudios (Jensen et al., 2000) y un estudio local (Valdivia, 1999), que las bacterinas comerciales de dos dosis presentan ventajas comparativas en ganancia de peso sobre los grupos control. Es posible que la escasa respuesta biológica se haya debido a la permanencia de los lechones vacunados cerca de los animales controles con el consiguiente aumento de exposición al patógeno y que se haya trabajado con una infección natural (Dawson et al., 2002b).

Se observó una menor cantidad de animales $(\mathrm{p}<0.05)$ del grupo inmunizado con algún grado de consolidación pulmonar (20.7\%) en relación con los animales del grupo control $(79.3 \%)$, lo que coincide con otros trabajos (Siugzdaite y Garlaite, 2002; Llopart et al., 2002; Dawson et al., 2002b); sin embargo, la reducción en el porcentaje de lesiones pulmonares fue mayor $(79.3 \%)$ que en otros estudios (Goodwin, 1984: 66\%; Hannan et al., 1997: 57\%; Dawson et al., 2002a: 50-60\%; Thacker y Thacker, 2001). Se tiene información que el efecto es mayor cuando la vacunación se realiza a las 6 semanas de edad en comparación con vacunaciones tempranas (Scheidt et al., 1990).

A pesar que en este estudio no se hizo un análisis sobre el impacto económico de la vacunación, se puede inferir que la disminu- 


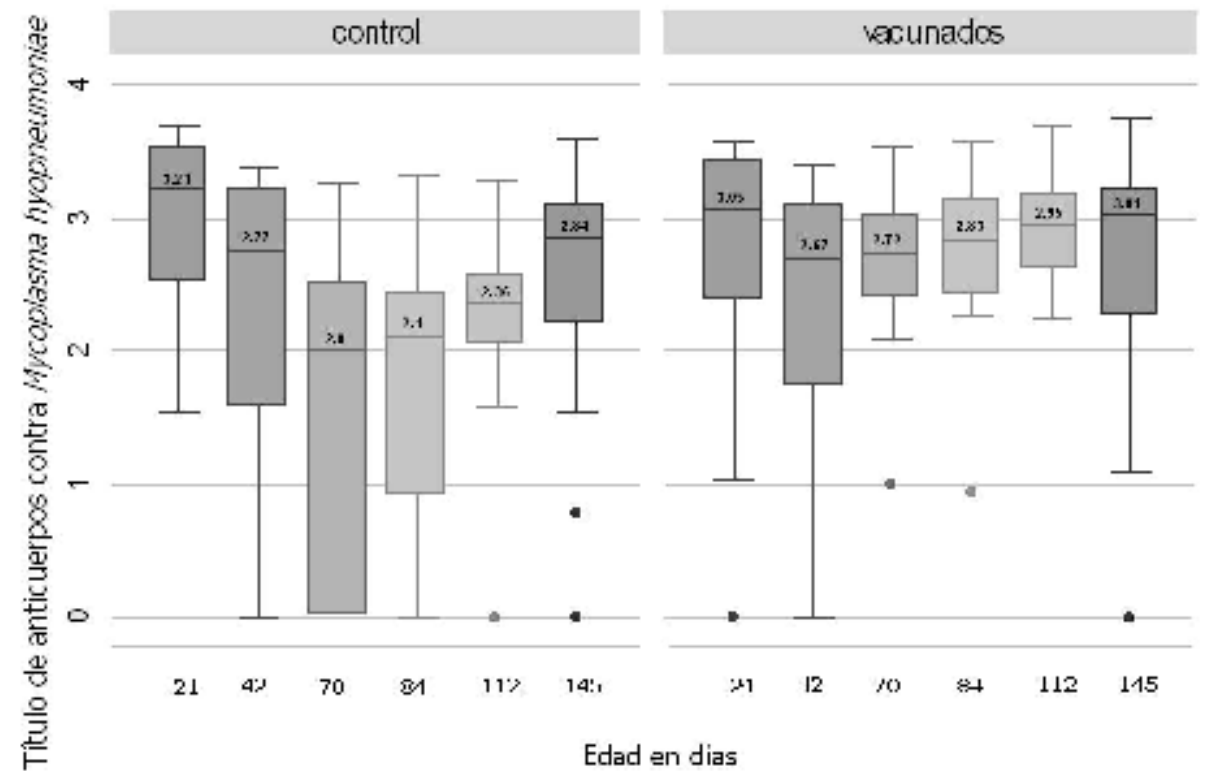

Figura 1. Mediana de los títulos de anticuerpos contra Mycoplasma hyopneumoniae en lechones que fueron inmunizados a los 42 días de edad o quedaron como controles, y cuyas madres fueron inmunizadas a los 85 días de gestación

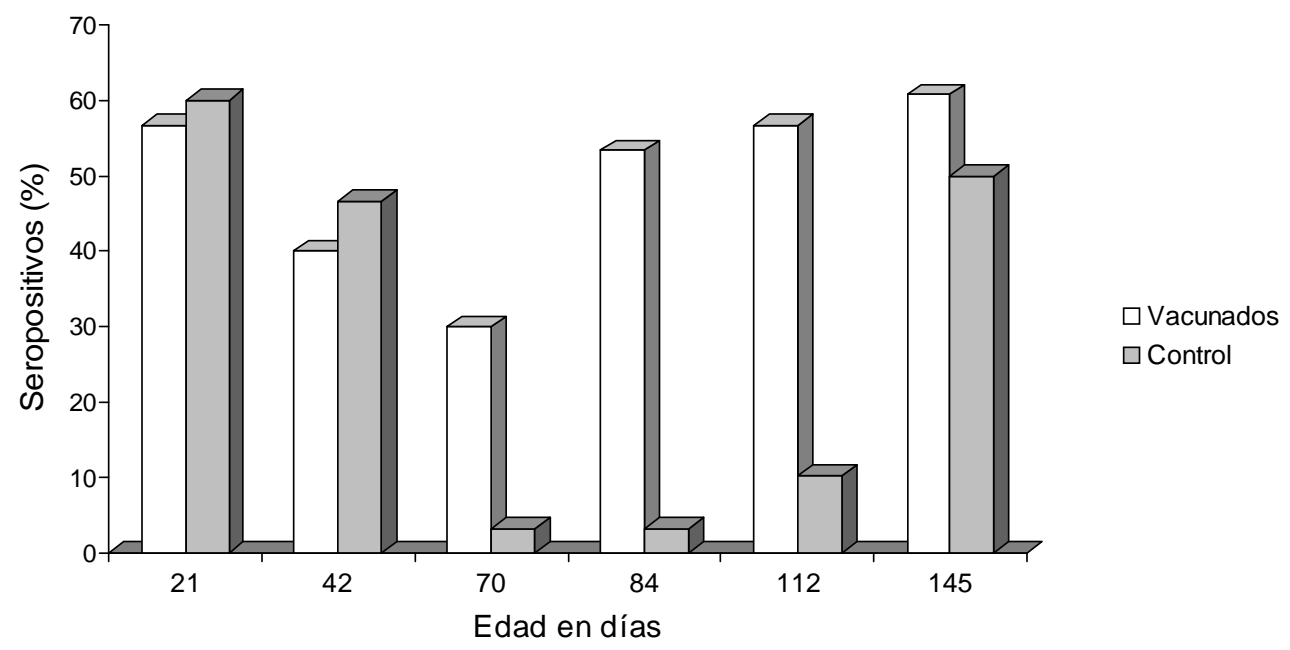

Figura 2. Frecuencia (\%) de lechones seropositivos a Mycoplasma hyopneumoniae en la prueba de ELISA indirecta, que fueron inmunizados a los 42 días de edad o quedaron como controles, y cuyas madres fueron inmunizadas a los 85 días de gestación

ción en los costos de mano de obra para aplicar una sola dosis, la reducción en las lesiones pulmonares, la mayor ganancia de peso y el ahorro en antibióticos, son claros indicios de las ventajas económicas del programa de inmunización bajo estudio.

\section{Conclusiones}

- La inmunización de lechones con bacterinas de dosis única contra Mycoplasma hyopneumoniae a los 42 
días de edad tuvo un efecto positivo en la respuesta inmune, redujo sustancialmente el porcentaje de consolidación pulmonar, pero no mejoró la ganancia de peso entre el destete y la edad de beneficio.

- La seroconversión en el grupo control ocurrió a las 16 semanas de edad, lo que coincide con el inicio de la etapa de engorde.

\section{Literatura Citada}

1. Blood, D.C.; O. Radostits; K. Hincheliff; C. Gay. 1999. Medicina Veterinaria. 9a ed. p 1195-1204. Ed. Interamericana McGraw Hill. Madrid.

2. Calle, E.S.; S.C. Camacho; A.M. Torres; P.N. Falcón; C.M. Cerón; R.E. Zacarías. 2003. Inmunidad natural e inducida contra Mycoplasma hyopneumoniae medida desde el nacimiento hasta la edad de mercado en cerdos bajo crianza tecnificada. Mundo Avícola y Porcino 44: 48-49.

3. Calsamiglia, M.; C. Pijoan; G.J. Bosch. 1999. Profiling Mycoplasma hyopneumoniae in farms using serology and a nested PCR technique. Swine Health Prod. 7: 263-268.

4. Clark, K. 1999. Mycoplasma hyopneumoniae: serology/vaccinology. Proc. Am. Assoc. Swine Practitioners p. 339343. St. Louis, USA.

5. Dawson, A; S. Thevasagayam; J. Sherington; J.D. Mackinnon; L.P. Stipkovits; A.R. Peters. 2002a. The duration of immunity after vaccination with a single dose Mycoplasma hyopneumoniae vaccine and the effect of serological status on lung lesion scores. Pig J. 50: 83-92.

6. Dawson, A; R.E. Harvey; S.J. Thevasagayam; J. Sherington; A.R. Peters. 2002b. Studies of the field efficacy and safety of a single dose Mycoplasma hyopenumoniae vaccine for pigs. Vet. Rec. 2: 535-538.
7. Goodwin, R.F.W. 1984. Apparent reinfection of enzootic pneumonia-free pig herds: early sign and incubation period. Vet. Rec. 115: 320-324.

8. Goodwin, R.F.W. 1985. Apparent reinfection of enzootic pneumonia-free pig herds: search for possible causes. Vet. Rec. 116: 690-694.

9. Hannan, P.C.T; H.M. Windsor; P.H. Ripley. 1997. In vitro susceptibilities of recent field isolates of Mycoplasma hyopneumoniae and Mycoplasma hyosynoviae to valnemulin (Econor ${ }^{\circledR}$ ), tiamulin and enrofloxacin and the in vitro development of resistance to certain antimicrobial agents in Mycoplasma hyopneumoniae. Res. Vet. Sci. 63: 157160.

10. Jayappa, H.; R. David; V. RappGabrielson; T. Wasmoen; E. Thacker; B. Thacker. 2001. Evaluation of the efficacy of Mycoplasma hyopneumoniae bacterin following immunization of young pigs in the presence of varing levels of maternal antibodies. Proc. Am. Assoc. Swine Vet. p 237-241. Nashville, USA.

11. Jensen, C.S; A.K. Ersbolla; J.P. Nielson. 2000. A meta-analysis comparing the effects of vaccines against Mycoplasma hyopneumoniae on daily weight gain. Proc. 9th Int. Soc. Vet. Epid. \& Econ. p 641-643. Breckenridge, Colorado, USA.

12. Joo, H.S; D.K. Suh; S. Rutten. 1988. Evaluation of control protocols for Mycoplasma hyopneumoniae infection in swine farms. MPPA Research Final report. St. Paul, Minnesota. Disponible en http://www.pork.org/porscience/ documents/evaluationofcontrol.pdf

13. Llopart, D.; J. Clota; R. March; I. Navarra; P. Riera; C. Artigas. 2002. Evaluation of the efficacy of a single dose Mycoplasma hyopneumoniae vaccination programme in challenged pigs. Pig J. 50: 8-27.

14. Morrison, R.B.; C. Pijoan; H.D. Hilley; V. Rapp. 1985. Microorganism associated with pneumonia in slaughter 
weight swine. Can. J. Comp. Med. 49: 129-137.

15. Piffer, I.A.; R.F. 1984. Effect of age on susceptibility of pigs to Mycoplasma hyopneumoniae pneumonia. Am. J. Vet. Res. 45: 478-481.

16. Pommier, P.; A. Keita; E. Pagot; J.R. Walters; A. Flochlay. 2000. Field efficacy of a Mycoplasma hyopneumoniae vaccine in the control of enzootic pneumonia in swine. Rev. Sci. Med. 151: 835-840.

17. Quinlan, J. 1998. Delayed vaccination. Pig Internat. 28: 5-29.

18. Ross, R.F. 2000. Enfermedades micoplásmaticas. En: Enfermedades del cerdo. $8^{\mathrm{a}}$ ed. Vol. 1. Cap 31. p 495-509. Ed. Interamericana. Buenos Aires, Argentina.

19. Scheidt, A.; V.B Mayrose; M.A. Hill. 1990. Relationship of growth performance to pneumoniae and atrophic rhinitis detected in pigs at slaugther. J. Am. Vet. Med. Assoc. 196: 881-884.

20. Siugzdaite, J.; K. Garlaite. 2002. Effect of vaccination against Mycoplasma hyopneumoniae in a pig herd from birth to slaughter. Acta Vet. Brno 71: 549-553.

21. Snedecor, G.W.; W.G Cochran. 1986. Statistical methods. $7^{\text {a }}$ ed. Iowa State University Press. 503 p.

22. Thacker, B.J.; T. Boettcher; T. Anderson; E. Thacker; T. Young. 1998. The influence of passive immunity on serological responses to Mycoplasma hyopneumoniae vaccination. Proc $15^{\text {th }}$ Int. Pig Vet. Soc. p 154. Birmingham, UK.

23. Thacker, B.J.; E.L. Thacker. 2001. Influence of maternally derived antibodies on the efficacy of a
Mycoplasma hyopneumoniae bacterin. Proc. Am. Assoc. Swine Veterinarians. p 513-515. Nashville, USA.

24. Thacker, E.; P. Halbur; R. Ross; R. Thanawongnuwech; B. Thacker. 1999. Mycoplasma hyopneumoniae potentiation of Porcine Reproductive and Respiratory Syndrome Virus-Induced Pneumonia. J. Clin. Microbiol. 37: 620627.

25. Thacker, E.L.; B.J. Thacker; M. Kuhn; P.A. Hawkins; W.R. Waters. 2000. Evaluation of local and systemic immune responses induced by intramuscular injection of a Mycoplasma hyopneumoniae bacterin to pigs. Am. J. Vet. Res. 61: 1384-1389.

26. Thacker, E.L. 2001. Mycoplasma diagnosis and inmunity. Proc. Am. Assoc. Swine Vet. p 467-469. Nashville, USA.

27. Torres, M. 2003. Determinación serológica de la infección con Mycoplasma hyopneumoniae en una granja de cerdos de crianza intensiva. Tesis de Médico Veterinario. Facultad de Medicina Veterinaria, Univ. Nacional Mayor de San Marcos. Lima. 51 p.

28. Valdivia, A.L. 1999. Respuesta a la vacunación contra neumonía enzoótica porcina en términos de producción en la explotación intensiva de cerdos. Tesis de Médico Veterinario. Facultad de Medicina Veterinaria, Univ. Nacional Mayor de San Marcos. Lima. 32 p.

29. Yagihashi, T.; T. Nunoya; T. Mitui; M. Tajima. 1984. Effect of Mycoplasma hyopneumoniae infection on the development of Haemophilus pleuroneumoniae pneumonia in pigs. Jpn. J. Vet. Sci. 46: 705-713. 